Sharif University of Technology
Scientia Iranica
Transactions E: Industrial Engineering
wCIENTIA

\title{
Minimizing maximum earliness in single-machine scheduling with flexible maintenance time
}

\author{
F. Ganji ${ }^{\mathrm{a}}$, Gh. Moslehi ${ }^{\mathrm{b}}$ and B. Ghalebsaz Jeddic,* \\ a. Department of Industrial Engineering, Golpayegan University of Technology, Golpayegan, P.O. Box 87717-65651, Iran. \\ b. Department of Industrial and Systems Engineering, Isfahan University of Technology, Isfahan, P. O. Box 84156-83111, Iran. \\ c. Faculty of Engineering, Department of Industrial Engineering, Urmia University, Urmia, P.O. Box 57561-15311, Iran.
}

Received 15 August 2015; received in revised form 15 April 2016; accepted 28 June 2016

\section{KEYWORDS}

Scheduling;

Flexible maintenance;

Branch-and-bound;

Earliness.

\begin{abstract}
We consider minimizing the maximum earliness in the single-machine scheduling problem with flexible maintenance. In this problem, preemptive operations are not allowed, the machine should be shut down to perform maintenance, tool changing or resetting takes a constant time, and the time window inside which maintenance should be performed is predefined. We show that the problem is NP-hard. Afterward, we propose some dominance properties and an efficient heuristic method to solve the problem. Also, we propose a branch-and-bound algorithm, in which our heuristic method, the lower bound, and the dominance properties are incorporated. The algorithm is computationally examined using 3,840 instances up to 14,000 jobs. The results impressively show that the proposed heuristic algorithm obtains the optimal solution in about $99.5 \%$ of the cases using an ordinary processor in a matter of seconds at most.

(C) 2017 Sharif University of Technology. All rights reserved.
\end{abstract}

\section{Introduction}

Nowadays, scheduling problems with availability constraints have found vast applications in many production and service systems. Machines and other resources may often be unavailable during the scheduling horizon due to breakdown, preventive maintenance, etc. in some time periods. Deterministic machine unavailability problems fall into four categories of: fixed unavailability constraint, periodic unavailability constraint, flexible maintenance (the subject of our study here), and periodic flexible maintenance. In all of these problems, it is assumed that the length of unavailability period or maintenance is known in advance. In scheduling problems, with one or periodic

*. Corresponding author. Tel.: +9844327r 5660;

Fax: +984432773591

E-mail addresses: ganji@gut.ac.ir (F. Ganji);

moslehi@cc.iut.ac.ir (G. Moslehi); b.jeddi@urmia.ac.ir (B.

Ghalebsaz Jeddi) fixed unavailability constraints, it is assumed that the starting time of the unavailability period or periods is determined in advance, but in scheduling problems with a flexible maintenance, the starting time of maintenance(s) is(are) a decision variable(s).

With regard to the single-machine scheduling problems with fixed single-period unavailability, Adiri et al. [1] proved that, for the objective of minimizing the total completion time, this problem (i.e. $1, h_{1} \| \Sigma C_{i}$ ) is NP-hard. Lee and Liman [2] showed that the SPT (Shortest Processing Time) rule for this problem has a tight worst-case error bound of $2 / 7$. Sadfi et al. [3] proposed an improved version of the SPT rule, called Modified SPT (MSPT), for the same problem, and they proved that the worst-case error bound for their MSPT algorithm is $3 / 7$. Kacem and Chu [4] aimed to minimize the weighted sum of completion times for the problem (i.e. $1, h_{1} \| \Sigma w_{i} C_{i}$ ) and proposed a branch-and-bound algorithm based on a set of improved lower bounds and heuristics. They claimed that their improved algorithm is able to solve instances 
of 6000 jobs in a reasonable amount of computation time.

Kacem et al. [5] developed a Mixed Integer Programming (MIP) model for the problem with the objective of minimizing the total completion time (i.e., $\left.1, h_{1} \| \Sigma C_{i}\right)$ and used two methods of dynamic programming and a branch-and-bound algorithm. Molaee [6] studied the problem with other separate objectives of minimizing the maximum earliness and minimizing the number of tardy jobs (respectively denoted by $1, h_{1} \| E_{\max }$ and $1, h_{1} \| \Sigma U_{i}$ ). They proposed a heuristic algorithm and an exact branch-and-bound method to solve $1, h_{1} \| E_{\max }$ after showing that the problem is NPhard. By proving a number of theorems and lemmas, they developed a lower bound and some efficient dominance rules, and so presented heuristic algorithm with $O(n \log (n))$ which was additionally used to calculate the upper bound. Computational results for 2400 instances showed that the branch-and-bound procedure is capable of optimally solving $98.79 \%$ of the instances. Then, for $1, h_{1} \| \Sigma U_{i}$ problem, by proving a number of theorems, they developed a heuristic procedure to solve the problem. They also proposed a branch-andbound approach which includes efficient upper and lower bounds and dominance rules. They claimed that "computational results for 2400 problem instances show that the branch-and-bound approach is capable of optimally solving $97.4 \%$ of the instances. The proposed heuristic procedure is then evaluated for the problems with large sizes, and it is observed that this procedure has good performance to solve these problems. Results also indicate that the proposed approaches are more efficient when compared to other methods" [6].

Later on, Molaee et al. [7] considered the objectives of reference [6] simultaneously (i.e. a bi-criterion objective to simultaneously minimize maximum earliness and number of tardy jobs), and they proposed a mathematical optimization model and a branch-andbound algorithm to solve it.

As for the second group of single-machine scheduling problems where we deal with two or more fixed periods of unavailability, Liao and Chen [8] considered minimizing the maximum tardiness (i.e. $1, h_{i} \| T_{\max }$ ) by providing a heuristic algorithm with $O\left(n^{2} \Sigma p_{i}\right)$ complexity and also by using a branch-and-bound method. Ji et al. [9] considered minimizing the makespan for this class of problems (i.e. $1, h_{i} \| C_{\max }$ ), and they proved that the worst case ratio of the classical LPT (Longest Processing Time) algorithm is 2. Chen [10] studied this problem to minimize the number of tardy jobs (i.e. $1, h_{i} \| \Sigma U_{i}$ ), and he proposed a branch-and-bound algorithm as well as a heuristic algorithm to solve it with complexity of $O\left(n^{2} \Sigma p_{i}\right)$.

The focus of this study is on the single-machine scheduling problem with flexible unavailability constraint (the previously mentioned third group of scheduling problems). Yang et al. [11] proved that solving the problem to minimize makespan is NP-hard, and they proposed a heuristic algorithm to solve it with complexity of $O(n \log (n))$. Chen [12] studied this problem to minimize the total tardiness (i.e., $\left.1, h_{1}|f a| \Sigma T_{i}\right)$ and proposed two mixed Binary Integer Programming (BIP) models to solve it. Also, Chen [13] proposed two mixed BIP models for this problem with the objective of minimizing the makespan (i.e., $\left.1, h_{1}|f a| T_{\max }\right)$. In another work, Chen [14] developed two mixed BIP models for solving $1, h_{1} \mid$ fa $\mid \bar{F}$ problem to minimize average flow time $\bar{F}$ for two cases of preemptive (i.e., job splitting is allowed) and nonpreemptive jobs.

As for the fourth group of the aforementioned problems, Low et al. [15] considered the single-machine scheduling problem with flexible periodic maintenance to minimize the makespan (i.e., $1, h_{i}|\mathrm{fpa}| C_{\max }$, where fpa stands for flexible periodic activity/maintenance) and proposed a heuristic algorithm to address it. Qi [16] studied $1, h_{i}|\mathrm{fpa}| \Sigma C_{i}$ and $1, h_{i} \mid$ fpa $\mid L_{\max }$ problems for the objectives of minimizing the total completion time and maximum lateness, respectively, where the number and the starting time of unavailability constraint are decision variables. They showed that these problems are NP-hard. Sbihi and Varnier [17] presented a heuristic method for the single-machine scheduling problem with several maintenance periods. Specifically, two situations were investigated in their study: first, maintenance periods were periodically fixed (i.e. $1, h_{i} \mid p a T_{\max }$ ); second, maintenance periods were not fixed, but the maximum permitted continuous working time of the machine was fixed (i.e. $\left.1, h_{i}|\mathrm{fpa}| T_{\max }\right)$.

Few researchers have considered the objective of minimizing maximum or total earliness. Such objectives can be appropriate in industries like those producing deteriorative products whose earliness cost can be a major cost of the system. Valente [18] presented a heuristic algorithm for the single-machine scheduling problem to minimize the total weighted earliness (i.e. $1 \| \Sigma w_{i} E_{i}$ ). Moslehi and Mahnam [19] considered the problem of scheduling jobs on a single machine to minimize the sum of maximum earliness and tardiness (i.e. $1 \| \Sigma E T_{\max }$ ) using efficient lower and upper bounds and some dominance rules. They also utilized branch-and-bound algorithm for solving the problem. In a more recent study, Moslehi and Rohani [20] considered the single-machine scheduling problem to obtain the Pareto optima for minimizing three objectives of maximum tardiness, maximum earliness, and number of tardy jobs using the branch-andbound algorithm.

In this paper, we consider the single-machine scheduling problem with flexible maintenance (with constant duration), and we are to minimize maximum 
earliness, $E_{\max }$, among all earliness, $E_{i}$. It is assumed that there is a fixed period inside which maintenance shall be performed, but the starting time of maintenance is flexible (denoted by $f a$ ) and is a decision variable; unforced idle time is not allowed and the jobs are non-preemptive, so the problem is denoted as $1, h_{1}|f a| E_{\max }$ where $E_{\max }=\max _{1 \leq i \leq n}\left\{E_{i}\right\}$.

The remainder of the paper is organized as follows: Section 2 elaborates on the problem and presents some lemmas and theorems used to develop solution procedures later on. A heuristic algorithm and a branch-and-bound scheme are presented to solve the problem in Section 3. Section 4 presents numerical examples and computational results to analyze the performance of the heuristic and branch-and-bound algorithms. Section 5 provides the concluding remarks and directions for future studies.

\section{Problem properties}

\section{Notation}

Trying to keep it as close as to the notations in the literature (e.g., [21]), we use the following notations throughout the paper:

$J$

$n$

$p_{i}$

$d_{i}$

$u$

$v$

W

$w$

$C_{i}$

$E_{i}$

Si

$p(S i)$

$s_{i}$

$\sigma$

$\sigma^{\prime}$

$\sigma_{\text {before }}$

$\sigma_{\text {after }}$
Set of all jobs

Number of jobs

(Integer valued) processing time of job $i$

(Integer valued) due date of job $i$

The earliest maintenance starting time

The latest maintenance completion time

Maintenance interval, i.e. $W=v-u$

Fixed (integer valued) maintenance time

Completion time of job $i$

Earliness of job $i$ calculated as $E_{i}=\max \left\{0, d_{i}-C_{i}\right\}=\left(d_{i}-C_{i}\right)^{+}$

Partial sequence $i$

(Integer valued) processing time of all jobs in $S i$

Slack of job $i$ where $s_{i}=d_{i}-p_{i}$

Set of partial sequence consisting of arranged jobs

Set of non-arranged jobs (complementary set of $\sigma$ )

Group of arranged jobs before the maintenance

Group of arranged jobs after the maintenance whose sequence is known, but their starting time is unknown, and $\sigma=\sigma_{\text {before }} \cup \sigma_{\text {after }}$

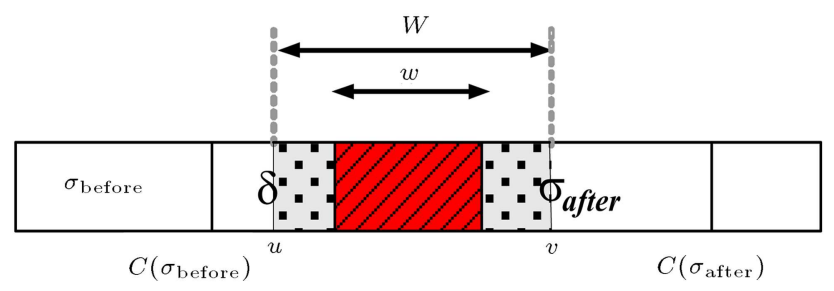

Figure 1. Notional form of the problem at hand.

$C(\cdot) \quad$ Completion time of any set of jobs, e.g. $\sigma_{\text {before }}$ or $\sigma_{\text {after }}$

$\delta \quad$ Immediate (and the only) idle time before the maintenance

$E_{i}(\cdot) \quad$ Earliness of job $i$ in any sequence

Figure 1 depicts the notations of problem $1, h_{1}|f a|$ $E_{\text {max }}$.

The decision variables are the sequence of the jobs, which also defines the starting time of any job and starting time of the maintenance operation.

Major assumptions of the study are as follows: All jobs are of single operation, and preemption or job splitting is not allowed (i.e. jobs are nonpreemptive), and they are simultaneously available at the beginning of the planning horizon; all data are integer including the processing times; unforced idle time is prohibited at any point including before the maintenance (i.e., it is not allowed to create an idle time that can be completely or partially occupied by a job; in other words, $\delta<\min \left\{p_{i} \mid i \in \sigma_{\text {after }}\right\}$ ); the period $[u, v]$, in which the maintenance should be performed, has been arranged in advance and clearly maintenance time, $w$, is smaller than $v-u$.

It is important to note that if an unforced idle time was allowed, all jobs could be processed after the maximum due date with an arbitrary arrangement so that the maximum earliness becomes zero although other criteria, which we do not consider here, may be hurt. However, it is not rational to create such idleness due to the highly valuable machine time.

Furthermore, in the problem at hand, all jobs cannot be processed before the maintenance (i.e., $\left.\Sigma p_{i}>v-w\right)$; otherwise, the problem reduces to the case of scheduling without maintenance, where the Minimum Slack Time (MST) sequence is the optimal solution as $d_{[1]}-p_{[1]} \leq d_{[2]}-p_{[2]} \leq \cdots \leq d_{[n]}-p_{[n]}$, where brackets indicate the rankings of the jobs in the sequence [22]. The problem $1, h_{1}|f a| E_{\max }$ has not been addressed in the literature. It is suitable to comment on the complexity of the problem at this point. Molaee [6] showed that the single-machine scheduling problem with a fixed unavailability constraint $\left(1, h_{1} \| E_{\max }\right)$ along with the assumption of allowed idle time is NP-hard. Thereby, in a particular situation when $w=v-u$, the problem $1, h_{1}|f a| E_{\max }$ converts to $1, h_{1} \| E_{\max }$, so the complexity of $1, h_{1}|f a| E_{\max }$ is at 
least as much as the complexity of $1, h_{1} \| E_{\max }$, so the problem at hand is NP-hard.

In this section, we present a few theorems and lemmas to establish two-solution procedures for the problem. Before discussing the theorems, note that the starting time of jobs in $\sigma_{\text {after }}$ is not fixed since we do not yet know when the maintenance will start and end; however, we will show that the MST rule is the basis (with some modification) for providing its optimal sequence. Also, note that the starting time of maintenance in this problem is a decision variable so that we cannot assume, by default, that maintenance service starts at first or ends at the last position of the maintenance time window (in that case, 1, $h_{1}|f a| E_{\max }$ converts to $\left.1, h_{1} \| E_{\max }\right)$.

Lemma 1: In problem 1, $h_{1}|f a| E_{\max }$, there is an optimal sequence where jobs in $\sigma_{\text {before }}$ and jobs in $\sigma_{\text {after }}$ are ordered according to MST rule.

Proof: We know that the MST rule is the optimal solution to $1 \| E_{\max }$ problem [22]. Jobs are divided into groups of 'before' and 'after' maintenance in this problem, where one of them starts processing at time zero (the beginning of the planning horizon) and the other starts right after maintenance. Therefore, each of them shall be ordered with respect to MST rule.

Based on this lemma, we introduce some methods here to solve $1, h_{1}|f a| E_{\max }$ problem. The MST sequence may generate a feasible sequence for $1, h_{1} \| E_{\max }$ and $1, h_{1} \mid$ fa $\mid E_{\max }$ problems; however, care should be taken because it is not necessarily optimal.

Dominance property 1: Sequences, in which the starting time of a job in $\sigma_{\text {before }}$ is not in $[u, v-w]$, are a dominant set for $1, h_{1} \mid$ fa $\mid E_{\max }$ problem.

Proof: Denote the last job in $\sigma_{\text {before }}$ by job $i$, and if its starting time is in period $[u, v-w]$ (and consequently, it is completed before $v-w$ ), then by changing the position of job $i$ and maintenance activity, the completion time of job $i$ increases; therefore, objective function, $E_{\max }$, will not get worse.

Theorem 1: In the problem $1, h_{1}|f a| E_{\max }, M S T$ sequence is optimal if it generates a feasible solution, no matter where the maintenance is located in $[u, v]$ window, and $E_{\max }$ is of a job from $\sigma_{\text {before }}$.

Proof: Denote a feasible solution, $S$, obtained by MST rule as $(S 1, i, S 2, w, S 3, S 4)$ where $E_{\max }$ is of job $i$ and $S j$ is a partial sequence of $S$, see Figure 2. We shall now show that no replacement decreases $E_{\text {max }}$.

By exchanging the positions of an arbitrary job from $S 1$ with an arbitrary job from $\sigma_{\text {after }}$ due to the

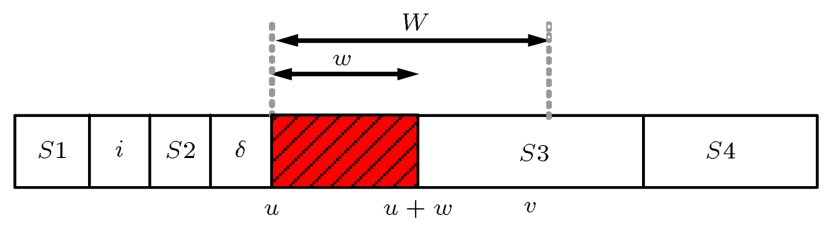

Figure 2. Sequence $S$ in Theorem 1.

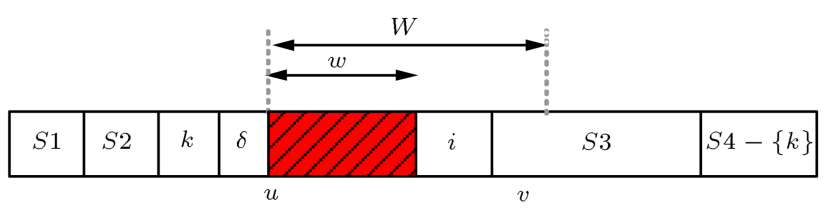

Figure 3. Sequence $S^{\prime}$ in Theorem 1.

regularity of MST rule in both $\sigma_{\text {before }}$ and $\sigma_{\text {after }}$, the replaced jobs are set in the first position of $\sigma_{\text {after }}$ and in the last position of $\sigma_{\text {before}}$, respectively. Thereby, the jobs in set $\sigma_{\text {before }}$ located after the replaced job in sequence $S$ are shifted to the left and their starting time decreases; therefore, $E_{\max }$ will not improve. Additionally, by changing the position of a job from $S 2$ and an arbitrary job from $\sigma_{\text {after }}, E_{\max }$ will not increase because the starting time of job $i$ has not changed. By exchanging job $i$ with an arbitrary job $k$ from $\sigma_{\text {after }}$, sequence $S^{\prime}$ as $(S 1, S 2, k, w, i, S 3, S 4-\{k\})$ is obtained, see Figure 3. Without loss of generality, it is assumed that job $k$ is located at the first position of $S 4$; two situations may occur: the partial sequence, $S 2$, is not empty, or it is empty.

Assume that partial sequence, $S 2$, is not empty and job $j$ is the first one in $S 2$. Then, the earliness values of jobs $i, j$, and $k$ in two sequences, $S$ and $S^{\prime}$, after switching jobs $k$ and $i$ are as follows:

$$
\begin{aligned}
& E_{i}(S)=\max \left\{\left(d_{i}-p(S 1)-p_{i}\right), 0\right\}, \\
& E_{k}\left(S^{\prime}\right)=\max \left\{\left(d_{k}-p(S 1)-p(S 2)-p_{k}\right), 0\right\},
\end{aligned}
$$

and:

$$
E_{j}\left(S^{\prime}\right)=\max \left\{\left(d_{j}-p(S 1)-p_{j}\right), 0\right\}
$$

where, by MST rule, we have $d_{i}-p i \leq d_{j}-p_{j}$. Thus, by comparing these relations, we conclude that $E_{i}(S) \leq$ $E_{j}\left(S^{\prime}\right)$.

Now, assume that partial sequence, $S 2$, is empty, so job $i$ is located in the last position of sequence $S$; thereby, regarding MST rule, we have $d_{i}-p_{i} \leq d_{k}-p_{k}$. By comparing the above relations, we arrive at $E_{i}(S) \leq$ $E_{k}\left(S^{\prime}\right)$.

So, the maximum earliness of sequence $S$ is not always greater than this value in sequence $S^{\prime}$, then by switching jobs $i$ and $k$ in sequence $S$, maximum earliness will not diminish.

Considering the possibility of shifting maintenance service back or forth in time, it might be possible to transfer a job from $\sigma_{\text {after }}$ to $\sigma_{\text {before }}$. 


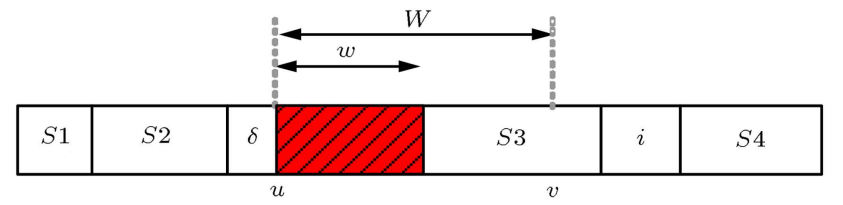

Figure 4. Sequence $S$ in Theorem 2.

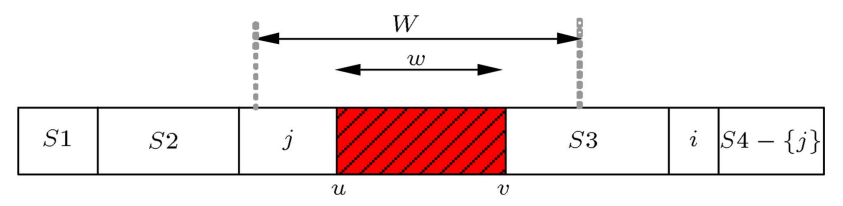

Figure 5. Sequence $S^{\prime}$ in Theorem 2.

Theorem 2: In MST sequence, for $1, h_{1} \mid$ fa $\mid E_{\max }$ problem, if $E_{\max }$ is of a job from $\sigma_{\text {after, }}$ then the objective function does not decrease by transferring a job from set $\sigma_{\text {after }}$ to $\sigma_{\text {before }}$.

Proof: The feasible solution, $S$, is generated by MST rule which is shown as $(S 1, S 2, w, S 3, i, S 4)$ and $E_{\max }$ is related to job $i$ (see Figure 4); regarding feasibility of sequence $S$, the starting time of jobs in set $\sigma_{\text {after }}$ decreases or does not change by transferring a job of set $S 3$ into set $\sigma_{\text {before }}$ due to filling the idle time before maintenance. Therefore, the objective function does not improve (does not decrease).

Also, by transferring job $i$ into $\sigma_{\text {before }}$ due to a decrease in the starting time of job $i$, the objective function does not improve. Now, assume that a job in $S 4$ is transferred to $\sigma_{\text {before }}$. Without loss of generality, assume that job $j$ is in the first position of $S 4$; hence, sequence $S^{\prime}$ is obtained as $(S 1, S 2, j, w, S 3, i, S 4-$ $\{j\})$, see Figure 5 .

Only job $j$ can affect the maximum earliness in sequence $S^{\prime}$, so earliness values of job $i$ in sequence $S$ and job $j$ in sequence $S^{\prime}$ are calculated according to the following equations:

$$
\begin{aligned}
E_{i}(S)= & \max \left\{\left(d_{i}-\max \left(C\left(\sigma_{\text {before }}, u\right)\right)-w\right.\right. \\
& \left.\left.-p(S 3)-p_{i}\right), 0\right\} \\
E_{j}\left(S^{\prime}\right)= & \max \left\{\left(d_{j}-p(S 1)-p(S 2)-p_{j}\right), 0\right\} .
\end{aligned}
$$

With regard to MST rule, we have $d_{i}-p_{i} \leq d_{j}-p_{j}$. Now, by comparing these relations, we obtain $E_{i}(S) \leq$ $E_{j}\left(S^{\prime}\right)$.

Therefore, transferring jobs from $\sigma_{\text {after }}$ to $\sigma_{\text {before }}$ does not improve the objective function.

Theorem 3: If the MST rule generates a feasible sequence for $1, h_{1}|f a| E_{\max }$ problem, and $E_{\max }$ corresponds to a job (e.g. job $i$ ) from $\sigma_{\text {after, }}$ then this sequence is optimal only with the following exception: when in the MST sequence, $p_{k}<p_{j}$ where $k=$

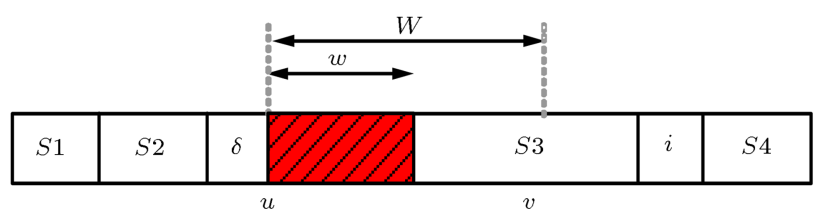

Figure 6. Sequence $S$ in Theorem 3.

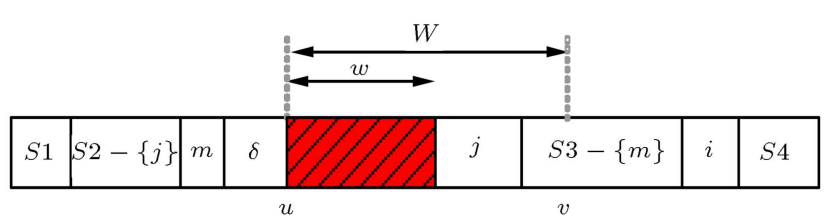

Figure 7. Sequence $S^{\prime}$ in Theorem 3 and the 2 nd condition.

$\operatorname{Arg} \min \{p\}$ and $j=\operatorname{Arg} \max \{p\}$, and exchanging jobs $k$ and $j$ causes the idle time before maintenance to become greater than zero (see Figure 6 for $S 3$ ).

Proof: Assume that $E_{\max }$ is of a job from $\sigma_{\text {after }}$, say job $i$. Thus, by switching the positions of an arbitrary job from $\sigma_{\text {before }}$ to an arbitrary job from $\sigma_{\text {after }}$, six cases may occur as follows (Figure 6 shows job $i$ in primary sequence $S$.):

1. It is obvious that pair-wise replacement of jobs in $\sigma_{\text {before }}$ (or $\sigma_{\text {after }}$ ) will not lead to any improvement in the objective function because the MST rule has not been met;

2. By exchanging the positions of jobs $m \in S 3$ and $j \in$ $\sigma_{\text {before }}$ with the assumption of $p_{m} \geq p_{j}$, sequence $S^{\prime}($ as $S 1, S 2-\{j\}, m, w, j, S 3-\{m\}, i, S 4)$ is obtained, see Figure 7. Due to regularity of MST rule in sets $\sigma_{\text {before }}$ and $\sigma_{\text {after }}$, jobs $j$ and $m$ should be located in the first position of $\sigma_{\text {after }}$ and in the last position of $\sigma_{\text {before }}$, respectively. The starting time of jobs in $\sigma_{\text {after }}$, namely job $i$, does not increase, and consequently $E_{\max }$ remains unchanged;

3. By exchanging the positions of jobs $m \in S 3$ and $j \in \sigma_{\text {before }}$ with the assumption $p_{m} \leq p_{j}$, if we have $\delta=0$ after replacement, then the starting time of job $i$ does not change; therefore, $E_{\max }$ will not decrease;

4. By exchanging the positions of job $i$ with an arbitrary job from $\sigma_{\text {before, }}$ the starting time of job $i$ decreases; therefore, the objective function will not improve;

5. By exchanging the positions of jobs $k \in S 4$ and $j \in \sigma_{\text {before }}$, we obtain sequence $S^{\prime}$ (as $S 1, S 2-\{j\}$, $k, w, j, S 3, i, S 4-\{k\})$, see Figure 8 .

Therefore, job $k$ shifts to the left and jobs in $\sigma_{\text {after }}$ may be shifted either to the right or left; in both cases, the following relations are true: 


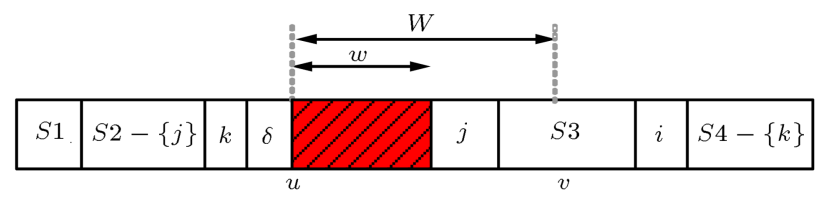

Figure 8. Sequence $S^{\prime}$ in Theorem 3 and the 5 th situation.

$$
\begin{aligned}
E_{i}(S)= & \max \left\{\left(d_{i}-\max \left(C\left(\sigma_{\text {before }}\right), u\right)-w\right.\right. \\
& \left.\left.-p(S 3)-p_{i}\right), 0\right\} \\
E_{k}\left(S^{\prime}\right)= & \max \left\{\left(d_{k}-p(S 1)-p(S 2)+p_{j}-p_{k}\right), 0\right\} .
\end{aligned}
$$

By comparing the above relations and taking that into account, due to MST, $d_{i}-p_{i} \leq d_{k}-p_{k}$, we obtain $E_{i}(S) \leq E_{k}\left(S^{\prime}\right)$. Consequently, the maximum earliness in sequence $S$ will not be greater than this value in sequence $S^{\prime}$.

6. By exchanging positions of job $m \in S 3$ and job $j \in \sigma_{\text {before }}$ under the assumption of $p_{m} \leq p_{j}$, the maximum earliness may be decreased if $\delta>0$.

Generally, in sequence $S$, by exchanging positions of an arbitrary job from $\sigma_{\text {after }}$ and a job from $\sigma_{\text {before, }}$, the maximum earliness will not improve in all situations except in situation (6). This concludes the Theorem.

This implies that the MST sequence is not always the optimal sequence for this problem.

The situations of Theorems 1 and 3 are summarized in Table 1.

Now, we present another theorem which provides us with the best possible value for the objective function (i.e., a lower bound), which will be helpful in recognizing the optimal solution.

Theorem 4: In $1, h_{1} \| E_{\max }$ problem when $h_{1}=v-u$ (i.e., the machine is unavailable in the whole period of $[u, v])$, if jobs are arranged based on MST rule where unforced idle time is allowed before maintenance, then its optimal solution is a lower bound for $1, h_{1} \mid$ fa $\mid E_{\max }$ problem.

Proof: Since the maximum earliness is not a regular objective function, it does not get worse if an unforced idle time is allowed. Thus, if maintenance is considered to cover the whole maintenance period $[u, v]$, then MST sequence can be a lower bound for $1, h 1|f a| E_{\max }$ problem.

Now, having these fundamental theorems, we present an efficient and quick heuristic method, which provides an optimal solution in a good majority of the cases and a well near-optimal solution in the rest of them. Further, we also present a modified branchand-bound procedure, which obtains the optimal solution.

\section{Problem solution}

\subsection{A Heuristic algorithm: Flexible MST}

In this section, we propose a heuristic algorithm (named Flexible MST or FMST) to tackle the problem at hand. Before discussing the algorithm in detail, it might be useful to provide a briefing of it in advance as follows:

- Order all jobs according to MST rule and fit the maintenance in a feasible place, and denote this sequence by $\sigma_{\mathrm{MST}}$. Using Theorems 1 and 3 , check if the optimality conditions are satisfied. If so, the problem is solved otherwise,

- Obtain the lower bound of the problem according to Theorem 4, and then check if any of the jobs from set $\sigma_{\text {after }}$ can be performed in the idle time before maintenance. If this is feasible, check if the objective function is equal to the calculated lower bound; if so, the optimal solution is obtained. Otherwise,

- The obtained sequence might be the optimal solution or just a near-optimal solution (which would be helpful as an upper bound for the branch-and-bound procedure, explained later in Section 3.2).

\subsubsection{The algorithm in detail}

The steps of FMST are as follows where asterisk sign $\left(^{*}\right)$ identifies the optimal value of any variable.

- Step 1: Let $J$ be the set of all jobs, i.e. $J=$ $\left\{J_{1}, J_{2}, \cdots, J_{n}\right\}$;

- Step 2: Order and index jobs according to MST rule, and then insert the maintenance operation in

Table 1. Optimal situations of MST sequence (Theorems 1 and 3).

\begin{tabular}{lcl}
\hline $\begin{array}{c}\text { Position of job } \\
\boldsymbol{i}=\operatorname{Arg} \max \{\boldsymbol{E}\}\end{array}$ & Theorem & \multicolumn{1}{c}{ Optimality conditions } \\
\hline$i \in \sigma_{\text {before }}$ & 1 & Always true \\
& & $\begin{array}{l}\text { Always true, except when } p_{k}<p_{j} \text { where } \\
i \in \sigma_{\text {after }}\end{array}$ \\
& 3 & $k=\underset{S 3}{\operatorname{Arg} \min }\{p\}$ and $j=\underset{\sigma_{\text {before }}}{\operatorname{Arg} \max }\{p\}$ and \\
& $\delta>0$ after replacement \\
\hline
\end{tabular}


between so that it begins at $u$. Denote this sequence by $\sigma_{\mathrm{MST}}$ and its objective function by $E_{\max -\mathrm{MST}}$;

- Step 3: Set $\sigma_{1}=\sigma_{\mathrm{MST}}$. Let $m$ be a counter for the number of scheduled jobs after maintenance and $m=1,2, \cdots, M$, where $M$ is the size of set $\sigma_{\text {after }}$, created firstly based on the MST schedule; so, initially, $m=1$. Let $i=\operatorname{Arg} \max \{E\}$. Calculate the idle time before maintenance $\delta=u-C\left(\sigma_{\text {before }}\right)$. Calculate a lower bound for $E_{\max }$ using Theorem 4;

- Step 4: Compare $\delta$ with $\min _{\sigma_{\text {after }}}\left\{p_{i}\right\}$. Then, we will face three situations:

a) If $\delta<\min _{\sigma_{\text {after }}}\left\{p_{i}\right\}$ and if $i=\operatorname{Arg} \max \{E\} \in \sigma_{\text {before }}$, then we have obtained the optimal solution based on Theorem 1 ; let $\sigma^{*}=\sigma_{1}$ and $E_{\max }^{*}=E_{\max }\left(\sigma_{1}\right)$ and exit;

b) If $\delta<\min _{\sigma_{\text {after }}}\left\{p_{i}\right\}$ and $i=\operatorname{Arg} \max \{E\} \in \sigma_{\text {after }}$, go to Step 5. But;

c) if $\delta>\min _{\sigma_{\text {after }}}\left\{p_{i}\right\}$ and if it is possible to switch the first job of $\sigma_{\text {after }}$ to the end of $\sigma_{\text {before }}$, do so and start Step 4 from the beginning; otherwise, if switching the first job of $\sigma_{\text {after }}$ to the end of $\sigma_{\text {before }}$ is not possible, let $m=m+1$ and go to Step 6, unless $m=M$; in that case, go to Step 11 to exit (Note that at the end of this step, a feasible sequence is obtained.);

- Step 5: Let $S 3$ be a subset of jobs in $\sigma_{\text {after }}$ with indices smaller than $i$ in MST sequence (see Figure 6). If $p_{k}<p_{j}$ where $k=\underset{S 3}{\operatorname{Arg} \min }\{p\}$ and $j=$ $\operatorname{Arg} \max \{p\}$, then, according to Theorem 3, swap $\sigma_{\text {before }}$

jobs $j$ with $k$, update $\sigma_{1}$ and $E_{\max }\left(\sigma_{1}\right)$, check if new $\delta \leq \min _{\sigma_{\text {after }}}\left\{p_{i}\right\}$, then go to Step 7 if so. Otherwise, the switching is not possible, and so the optimal solution is obtained; let $\sigma^{*}=\sigma_{1}$ and $E_{\max }^{*}=E_{\max }\left(\sigma_{1}\right)$ and exit;

- Step 6: By starting from job $m$ and checking every job forward, if transferring any job from $\sigma_{\text {after }}$ to $\sigma_{\text {before }}$ is possible, add that job into $\sigma_{\text {before }}$ according to MST; note that the other jobs are already in MST sequence. Update $\delta$. If $\delta \leq \min _{\sigma_{\text {after }}}\left\{p_{i}\right\}$, go to Step7; otherwise let $m=m+1$ and continue this process until all jobs in $\sigma_{\text {after }}$ are checked if they could be transferred to $\sigma_{\text {before; }}$

- Step 7: If $E_{\max }(\sigma)$ does not correspond to a job in $\sigma_{\text {before, }}$, then go to Step 8. Otherwise, if $E_{\max }(\sigma)$ corresponds to a job in $\sigma_{\text {before, }}$ then (because the MST sequence is now shuffled) do the following: Let $m=m+1$, start from job $m$ in $\sigma_{\text {after }}$, if its transferring to the end of $\sigma_{\text {before }}$ is possible, do so and transfer the job with maximum earliness in $\sigma_{\text {before }}$ into $\sigma_{\text {after }}$, slide maintenance forward if necessary and possible to fit the job into $\sigma_{\text {before }}$ Update sequences in $\sigma_{\text {before }}$ and $\sigma_{\text {after }}$ according to MST rule separately and calculate $\delta$.

By selecting and replacing all jobs in $\sigma_{\text {after }}$, the objective function is calculated, and the best sequence among them is selected. If $\delta \leq \min _{\sigma_{\text {after }}}\left\{p_{i}\right\}$, then go to Step 11 to exit; otherwise, let $m=m+1$ and go to Step 9;

- Step 8: Let $m=i+1$ where $i=\operatorname{Arg} \max \{E\}$ and go to Step 9;

- Step 9: If switching job $m$ to $\sigma_{\text {before }}$ is not possible, go to Step 10. Otherwise, add job $m$ (a job after $i$ ) to $\sigma_{\text {before}}$, and then let maintenance in an appropriate position and update the jobs in $\sigma_{\text {before }}, \sigma_{\text {after }}$ and update $\delta$. By selecting and replacing every job in $\sigma_{\text {after }}$, calculate the objective function and select the best sequence among them. If $\delta \leq \min _{\sigma_{\text {after }}}\left\{p_{i}\right\}$, go to Step 11 to exit; otherwise, let $m=m+1$ and repeat Step 9 from the beginning;

- Step 10: If $m=M$, the number of jobs in initial

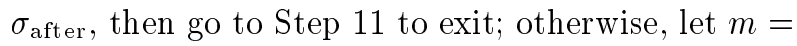
$m+1$ and go to Step 9 ;

- Step 11: If $E_{\max }\left(\sigma_{1}\right)$ is equal to the lower bound, then $\sigma_{1}$ is optimal, let $\sigma^{*}=\sigma_{1}$ and $E_{\max }^{*}=$ $E_{\max }\left(\sigma_{1}\right)$. Otherwise, a solution is obtained which still might be optimal or just near optimal (This will be checked by the branch-and-bound algorithm in Section 3.2.). Exit the algorithm.

\subsubsection{Computational complexity of FMST}

Based on the steps of FMST algorithm, its complexity at Step 2, in which the jobs are ordered according to MST rule, is $O(n \log (n))$. By proceeding in the algorithm and pair-wise replacement of the jobs in Steps 7 and 9 , the overall complexity of the proposed FMST is $O\left(n^{2}\right)$.

\subsection{A branch-and-bound procedure}

As shown in the final step of the FMST algorithm, in some cases, the conditions of Theorems 1 and 3 are not met nor the lower bound of Theorem 4 is reached; therefore, the algorithm could not obtain the optimal solution. In such cases, by having a feasible and nearoptimal solution from the FMST algorithm, we proceed with a branch-and-bound procedure to obtain an exact optimal solution for the problem.

\section{An upper bound}

The quantity of the objective function returned from execution of the FMST algorithm, which corresponds to a feasible and near-optimal solution, can be regarded as an upper bound for our minimization problem. Having this upper bound will expedite the procedure, and there is a need to calculate a lower bound in every step, as discussed below. 


\section{A lower bound}

Based on the following theorem, we generate a lower bound for partial sequences of the procedure obtained in every node.

Theorem 5: In partial sequence $\sigma$ (consisting of arranged jobs) for $1, h_{1} \mid$ fa $\mid E_{\max }$ problem, the lower bound is given by:

$$
L B(\sigma)=\max \left\{E_{\max }(\sigma), E_{\max }\left(\sigma^{\prime}\right)\right\},
$$

where $\sigma^{\prime}$ is the set of non-arranged jobs ordered according to MST rule and with the assumption that $h_{1}$ in $1, h_{1} \| E_{\max }$ problem covers the whole $[u, v]$ window, i.e., $h_{1}=v-u$.

Proof: It is clear that jobs in $\sigma^{\prime}$ with arbitrary order will not affect the objective function of partial sequence $\sigma$. In addition, arranging jobs in $\sigma^{\prime}$ based on MST rule in periods $\left[C\left(\sigma_{\text {before }}\right), u\right]$ and $[v, \infty)$, assuming that the unforced idle time is not allowed, yields the minimum value for the maximum earliness. Therefore, the objective function for complete sequence will not be smaller than the aggregate of two values $E_{\max }(\sigma)$ and $E_{\max }\left(\sigma^{\prime}\right)$.

The branch-and-bound procedure for the problem at hand is as a binary tree consisting nodes and branches, and each node generates two branches based on locating jobs before or after the maintenance window. At first, before starting the branch-and-bound procedure, jobs shall be ordered and indexed according to the MST rule. In every level, new branches are generated by adding a job from the MST list to the end of the previous jobs before the maintenance if possible (by sliding the maintenance back or force if necessary), or otherwise, to the end of the jobs after the maintenance. Thus, each node provides a partial sequence $(\sigma)$ for $\sigma_{\text {before }}$ and $\sigma_{\text {after }}$. The search strategy in this procedure is backtracking (namely, the procedure goes through every branch all the way until reaching a fathomed node before moving to another branch at the previous level). The branching continues until all unfathomed branches are visited, or the allowed processing time is over.

The fathoming of a node occurs in three conditions:

1. A complete schedule for all jobs is obtained;

2. Lower bound of the partial sequence, $L B(\sigma)$, calculated based on Theorem 5 , is greater than the upper bound;

3. A dominated partial sequence is obtained based on the Dominance Property 1.

In the first case, we update the upper bound if new $E_{\max }$ is less than the current upper bound.

\section{Numerical examples and results}

To evaluate the performance of the proposed branchand-bound and FMST algorithms, we code them in $\mathrm{C}^{++}$and carried out plenty of numerical experiments on a personal computer with a 3 GB RAM, Core 2Duo, CPU P8400, Pentium 4 under Windows 7 operating system. We generate parameters of test problems as used by Kacem, et al. [5], Yang, et al. [11], and Chen [13] as examples. Our experiment is performed using 16 different problem sizes of $n \in\{10,20,30$, 50, 100, 200, 300, 500, 700, 1000, 2000, 4000, 6000, $8000,12000,14000\}$. Processing times, $p$, are randomly chosen from a discrete uniform distribution over $[1,10]$, identically done in some other references, e.g. Sbihi and Varnier [17], Liao and Chen [8], and Pathumnakul and Egbelu [23]. Due dates $d$ are uniformly distributed over $\left[(1-C-Q / 2) \Sigma p_{i},(1-C+Q / 2) \Sigma p_{i}\right]$, subject to $d_{i} \geq p_{i}$, where $C$ and $Q$ take values of 0.2 and 0.6. The start of maintenance window, $u$, is taken as the integer part of $\frac{1}{4} \Sigma p_{i}, \frac{1}{2} \Sigma p_{i}$, and $\frac{3}{4} \Sigma p_{i}$, subject to $u \geq \max _{1 \leq i \leq n}\left\{p_{i}\right\}$, while the end of maintenance window is $v=u+30$. Maintenance times $w$ are all randomly chosen over $w \in[1,15]$ and $[16,30]$, where $v-u \geq w$.

For any problem with $n$ jobs, we run 10 random sampling (replications) for each combination of parameters. Therefore, a total of $3840(=10 \times 16 \times$ $2 \times 2 \times 3 \times 2$ ) test problems are examined in our study. For solving these problems, 3600 seconds of time constraint is applied. Table 2 presents the results, where the column "Num-opt" shows the number of problems that is solved optimally by branch-and-bound or FMST algorithms in less than one hour. Subcolumn "FMST" shows the number of problems that is solved optimally by FMST algorithm composed of two parts of "MST" and "Total". In the MST column, the number of optimally solved problems using Theorems 1 and 3 and the number of optimally solved problems in the "Total" column using the heuristic algorithm are presented. The next subcolumn, titled "BB", shows the number of problems that is solved optimally by branch-and-bound algorithm. Column "Cutting nodes \%" shows the average number of cut nodes (because of lower bound and dominance property) relative to all traveled nodes in percent. " $0.00 "$ in the lower bound means that the associated problems have reached the optimal solution merely by cutting the first nodes in less than 0.005 seconds, so they are rounded to 0.00 .

Applying the Dominance Property 1 in the structure of branch-and-bound algorithm helps to discard plenty of non-optimal solutions, and consequently the percentage of cutting nodes decreases dramatically. As shown in Table 2, in the series of odd numbers, all the problems were solved optimally without entering 
Table 2. Performance of the branch-and-bound and FMST algorithms in 24 series.

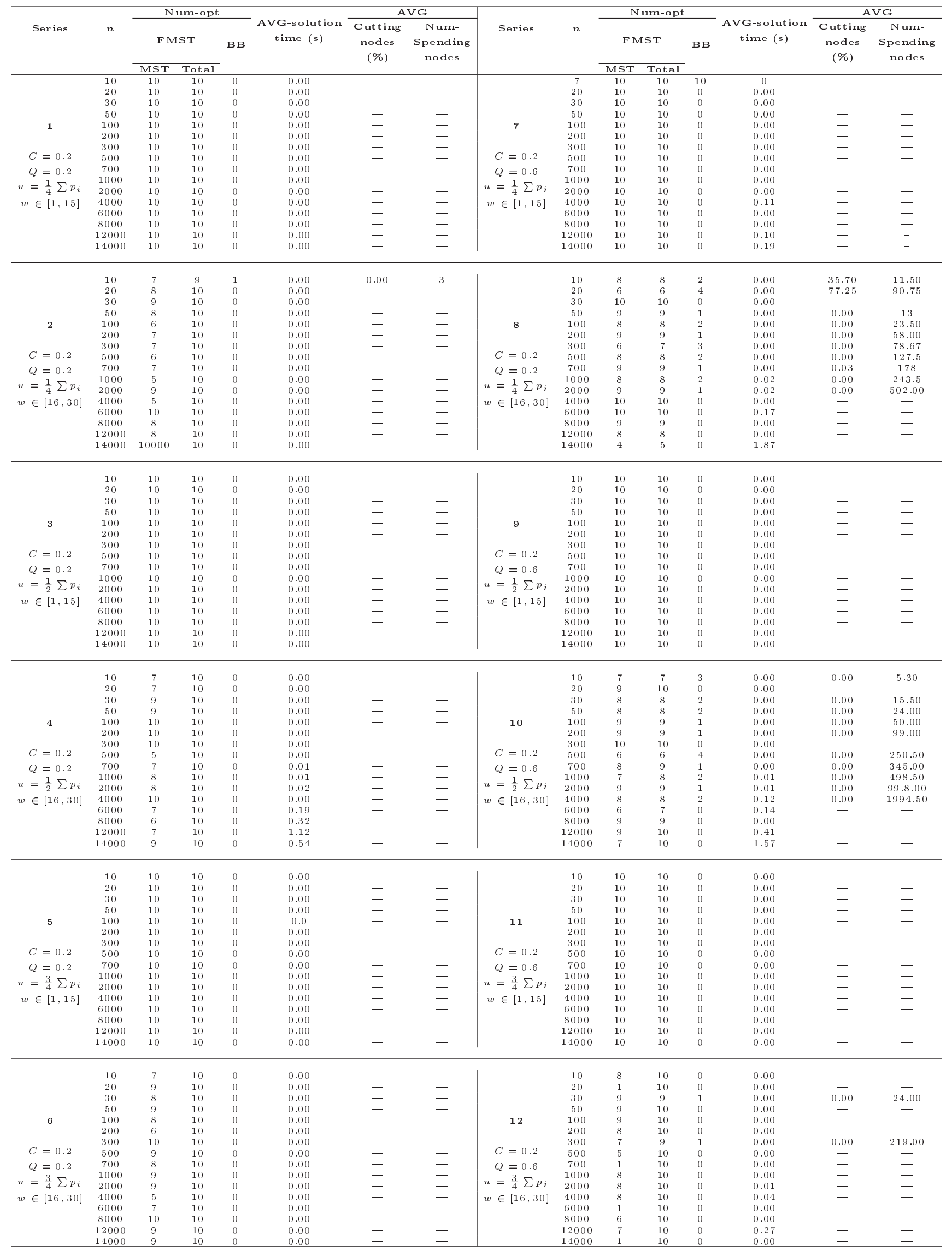


Table 2. Performance of the branch-and-bound and FMST algorithms in 24 series (continued).

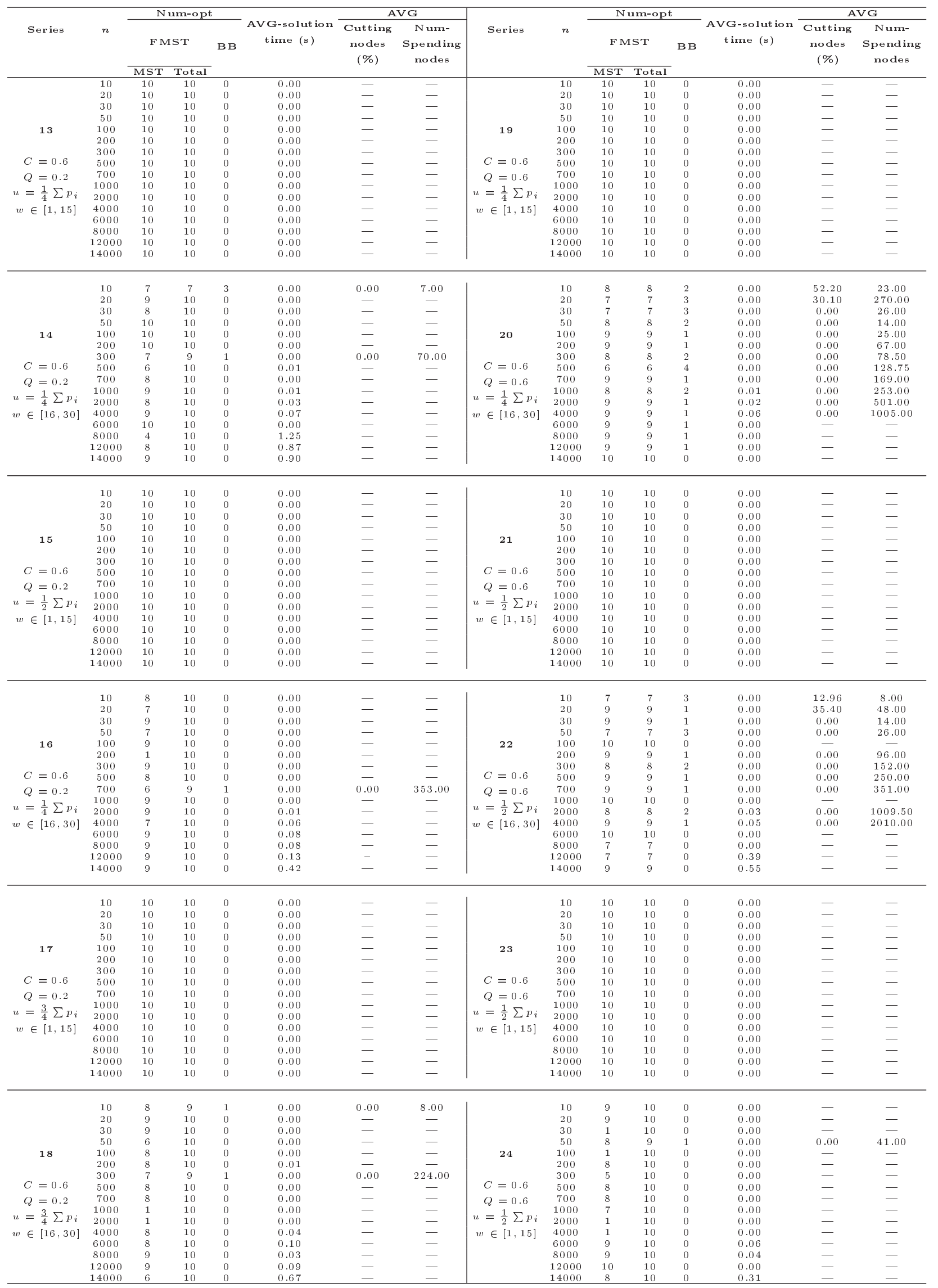


the branch-and-bound algorithm, and also satisfactory results were obtained for the rest of the series. Among these 24 series, $99.48 \%$ (i.e., 3820 out of 3840 ) of the problems were solved optimally in a matter of a couple of seconds at most.

In the series of odd numbers, the ability of FMST algorithm to obtain the optimal solution is very high. This might be due to their short-time maintenance activity, and consequently high flotation in these series. Larger $u$ in even series leads to decreasing the number of early jobs in $\sigma_{\text {after }}$ at the optimal solution, and very likely, the maximum earliness is obtained from the jobs in $\sigma_{\text {before }}$. Thereby, for large $u$, it is likely that in MST sequence, the job with maximum earliness is located before the maintenance. Thus, due to the large value of $u$, the number of problems that is optimal based on Theorem 1 increases, and also probability of effect of switching a job from $\sigma_{\text {after }}$ to $\sigma_{\text {before }}$ on maximum earliness decreases.

In series $2,8,14$, and 20 where $u$ possesses the least value, $81.87 \%$ of 640 problems were solved optimally by Theorem $1,10.81 \%$ by FMST algorithm, and $7.31 \%$ by branch-and-bound algorithm. In these series, $1.71 \%$ of the problems were left unsolved. It is seen in Figure 9 that the performance of the heuristic algorithm is higher for larger $u$. So, in series $6,12,18$, and 24 where $u$ has the highest value, efficiency of the heuristic algorithm has taken its highest value; in these series, it can solve $99.21 \%$ of the problems optimally, and the proportion of the times used by the branch-and-bound procedure is $0.78 \%$.

From Table 2, we also observe that the solution time, among the problems whose optimal solutions were achieved, has minor changes in terms of $u$ (see the series $1,2,7,8,13,14,19,20$ for $u=\frac{1}{4} \Sigma p_{i}$, series $3,4,9,10,15,16,21$, and 22 for $u=\frac{1}{2} \Sigma p_{i}$, and series $5,6,11,1217,18,23$, and 24 for $\left.u=\frac{3}{4} \Sigma p_{i}\right)$.

It is important to further observe how changes in $w$ affect the time until reaching the optimal solution. According to Table 2, in the series of odd numbers, (i.e., $1,3, \cdots, 23)$ where $w$ is in $[1,15]$, all problems were solved in less than 0.005 seconds on average on

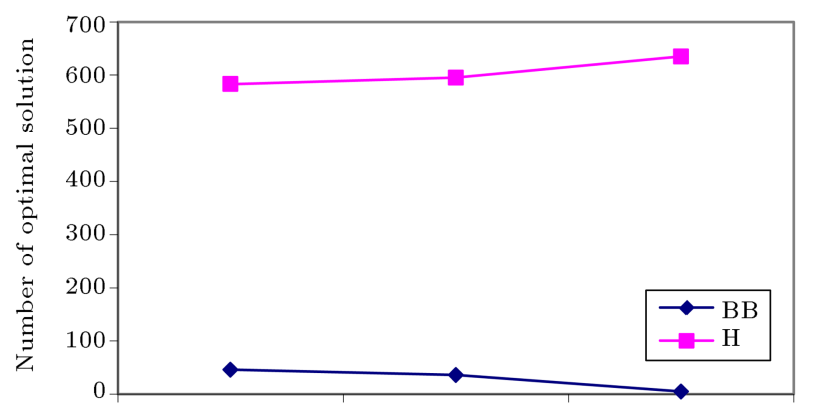

Figure 9. Performances of branch-and-bound and FMST algorithms in terms of $u$. all problems in each set. On the other hand, in the series of even numbers, (i.e., $2,4, \cdots, 24$ ) where $w$ is in $[16,30]$, six of these problem sets were solved in less than 0.005 seconds on average on all problems in each set, and six other series were solved between 0.006 and 0.025 seconds. We note that the processing time is not meaningfully sensitive to such changes in $w$.

Analyzing the performance of FMST and branchand-bound algorithms in terms of the range of due dates or $Q$ shows that increasing $Q$ expands upper bound for due date; hence, the number of early jobs in these problems increases. Even the maximum earliness can be related to the jobs in $\sigma_{\text {after }}$, and this fact results in going through more branches of the branch-andbound tree. For this reason, the performance of the heuristic algorithm is decreased by increasing $Q$. As it is depicted in Figure 10, in series 2, 4, 6, 14, 16, 18 of Table 2 where $Q=0.2$, the efficiency of heuristic algorithm is higher than that in series $8,10,12,20$, 22 , and 24 with $Q=0.6$. In the series of $Q=0.2$, the following fractions of the tested problems (out of 640 problems) are solved optimally: $71.83 \%$ using Theorem 1, 26.91\% using FMST algorithm, and 1.25\% using branch-and-bound algorithm. A tiny fraction of $0.16 \%$ of these problems was left unsolved using our methods. Also, in the series of $Q=0.6$, the following fractions of the test problems are solved optimally out of 640 problems: $76.97 \%$ by Theorem 1, $10.30 \%$ by FMST algorithm, and $12.72 \%$ by branch-and-bound algorithm. In these series, $2.97 \%$ of the problems were left unsolved.

Referring to Figure 10, it is concluded that by increasing $Q$, because the number of early jobs increases, more problems enter into the branch-andbound algorithm and more branches are visited. It is worth mentioning that the branch-and-bound algorithm subjected to time constraint in different series is not capable of solving some problems with the size of 6,000 jobs or more. Generally speaking, we observe that the problem series with higher values of $w$ and $Q$ and lower values of $u$ are more difficult to solve than the other series.

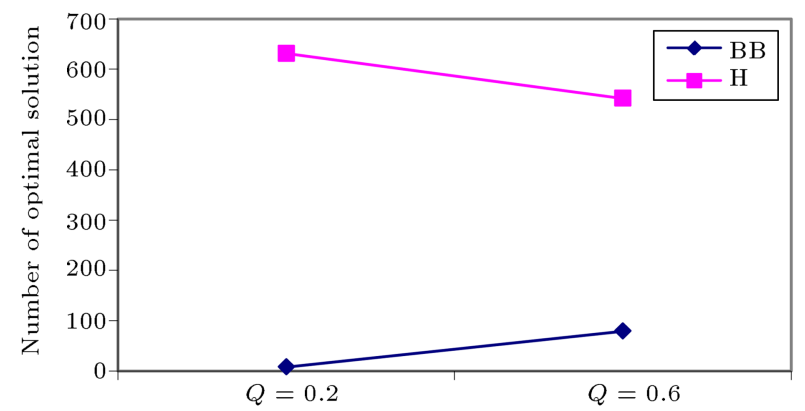

Figure 10. Performance of branch-and-bound and FMST algorithms in terms of $Q$. 


\section{Conclusion}

In this paper, the scheduling problem for a single machine with a flexible maintenance to minimize the maximum earliness was considered. In this problem, we let the starting time of maintenance be a decision variable inside a specified time window. All jobs were nonpreemptive and no unforced idle time was allowed. First, we showed that it is an NP-hard problem. Then, we proved several theorems and developed a heuristic algorithm (denoted by FMST) to solve it. Also, we proposed a branch-and-bound algorithm along with a lower bound and efficient dominance rule. In this approach, the FMST algorithm was applied as the upper bound. 3840 classic test problems in the form of 24 series were generated and solved using the aforementioned algorithms. Computational results demonstrated that $97.18 \%$ and $2.29 \%$ of the problems were solved optimally by FMST and the branch-andbound algorithms, respectively, at most in a matter of seconds; however, a tiny proportion of $0.52 \%$ of the problems could not be solved. Based on the results of standard test problems solved here, some sensitivity analyses on the performance of the proposed methods in terms of maintenance time, duration, and starting time of the allowed maintenance window were presented.

\section{References}

1. Adiri, I., Bruno, J., Frostig, E. and Rinnoy Kan, A.H.G. "Single machine flow-time scheduling with a single breakdown", Acta Information, 26, pp. 679-696 (1989).

2. Lee, C.Y. and Liman, S.D. "Single machine flowtime scheduling with scheduled maintenance", Acta Information, 29, pp. 375-382 (1992).

3. Sadfi, C., Penz, B., Rapine, C., Blazevicz, J. and Formanowicz, P. "An improved approximation algorithm for the single machine total completion time scheduling problem with availability constraints", European Journal of Operational Research, 161, pp. 3-10 (2005).

4. Kacem, I. and Chu, C. "Efficient branch-and-bound algorithm for minimizing the weighted sum of completion times on a single machine with one availability constraint", International Journal of Production Economics, 112, pp. 138-150 (2008).

5. Kacem, I., Chu, C. and Souissi, A. "Single-machine scheduling with an availability constraint to minimize the weighted sum of the completion times", Computers and Operations Research, 35, pp. 827-844 (2008).

6. Molaee, E. "Single machine scheduling problem with availability constraint", M.S Thesis, Department of Industrial and Systems Engineering, Isfahan University of Technology, Isfahan, Iran (2009).

7. Molaee, E., Moslehi, G. and Reisi, M. "Minimizing maximum earliness and number of tardy jobs in the single machine scheduling problem", Computers and Mathematics with Applications, 60, pp. 2909-2919 (2010).

8. Liao, C.J. and Chen, W.J. "Single-machine scheduling with periodic maintenance and nonresumable jobs", Computers and Operations Research, 30, pp. 13351347 (2003).

9. Ji, M., He, Y. and Cheng, T.C.E. "Single-machine scheduling with periodic maintenance to minimize makespan", Computers and Operations Research, 34, pp. 1764-1770 (2007).

10. Chen, W.J. "Minimizing number of tardy jobs on a single machine subject to periodic maintenance", Omega, 37, pp. 591-599 (2009).

11. Yang, D.L., Hung, C.L., Hsu, C.J. and Chen, M.S. "Minimizing the makespan in a single machine scheduling problem with a flexible maintenance", J. Chinese Inst. Insyst. Eng., 19, pp. 63-66 (2002).

12. Chen, J.S. "Optimization models for the machine scheduling problem with a single flexible maintenance activity", Engineering Optimization, 38, pp. 53-71 (2006).

13. Chen, J.S. "Scheduling of nonresumable jobs and flexible maintenance activities on a single machine to minimize makespan", European Journal of Operational Research, 190, pp. 90-102 (2008).

14. Chen, J.S. "Using integer programming to solve the machine scheduling problem with a flexible maintenance activity", Journal of Statistics and Management Systems, 9, pp. 87-104 (2006).

15. Low, C., Ji, M., Hsu, C.J. and Su, C.T. "Minimizing the makespan in a single machine scheduling problems with flexible and periodic maintenance", Applied Mathematical Modelling, 34(2), pp. 334-342 (2009).

16. Qi, X. "A note on worst-case performance of heuristics for maintenance scheduling problems", Discrete Applied Mathematics, 155, pp. 416-422 (2007).

17. Sbihi, M. and Varnier, C. "Single-machine scheduling with periodic and flexible periodic maintenance to minimize maximum tardiness", Computers and Industrial Engineering, 55, pp. 830-840 (2008).

18. Valente, J.M.S. "Local and global dominance conditions for the weighted earliness scheduling problem with no idle time", Computers and Industrial Engineering, 51, pp. 765-780 (2006).

19. Moslehi, G. and Mahnam, M. "A branch-and-bound algorithm to minimize the sum of maximum earliness and tardiness in the single machine", International Journal of Operational Research, 4, pp. 458-483 (2010).

20. Moslehi, G. and Rohani, M. "Finding Pareto optima for maximum tardiness, maximum earliness and number of tardy jobs", International Journal of Operational Research, 14(4), pp. 433-452 (2012).

21. Pinedo, M.L., Scheduling: Theory, Algorithms, and Systems, 4th Edn., Prentice Hall (2012). 
22. Baker, K.R. and Trietsch, D., Principals of Sequencing and Scheduling, John Wiley and Sons, Inc., New York (2009).

23. Pathumnakul, S. and Egbelu, P.J. "Algorithm for minimizing weighted earliness penalty in single-machine problem", European Journal of Operational Research, 161, pp. 780-796 (2005).

\section{Biographies}

Fatemeh Ganji is an Instructor at the Department of Industrial Engineering at Golpayegan University of Technology, Iran. She obtained her Bachelor's degree in Industrial Engineering from Golpayegan University of Technology, and Master's degree in Operations Research from Isfahan University of Technology, Iran. Her main lines of research are production planning, scheduling and sequencing, and design of industrial systems. Besides teaching and research, she has been involved in many projects in industry.

Ghasem Moslehi is an Associate Professor at the Department of Industrial and Systems Engineering, Isfahan University of Technology, Isfahan, Iran. He obtained a Bachelor's degree in Industrial Engineering, a Master's degree in Operations Research from Isfahan University of Technology, Iran, and a PhD in Industrial Engineering from Tarbiat Modarres University, Tehran, Iran. His main lines of research interests are scheduling and sequencing, production planning, and engineering economy. Dr. Moslehi has supervised many MSc and Doctorate thesis, published more than 100 refereed papers in those fields, and also participated in numerous international conferences.

Babak Ghalebsaz Jeddi is an Assistant Professor at the Faculty of Engineering, Urmia University, Iran. He previously lectured at Sharif University of Technology, Iran. He received his BSc degree in Industrial Engineering from Sharif University of Technology, Iran, and MSc degrees from Tehran University, Iran, and University of Cincinnati, Ohio. His $\mathrm{PhD}$ degree is conferred at George Mason University, Virginia, from the Department of Systems Engineering and Operations Research. His academic interests lie in applied side of operation research, systems engineering, statistics, and microeconomics in areas such as inventory and production control, air transportation system analysis, time series analysis and forecasting, quality control, and mobile network optimization. 